\title{
Assessing the Accuracy of Industrial Robots through Metrology for the Enhancement of Automated Non-Destructive Testing*
}

\author{
M. Morozov, J. Riise, R. Summan, S.G. Pierce, C. Mineo, C.N. MacLeod, R.H. Brown
}

\begin{abstract}
This work presents the study of the accuracy of an industrial robot, KUKA KR5 arc HW, used to perform quality inspections of components with complex shapes. Laser tracking and large volume photogrammetry were deployed to quantify both pose and dynamic path accuracies of the robot in accordance with ISO 9283:1998. The overall positioning pose inaccuracy of the robot is found to be almost $1 \mathrm{~mm}$ and path inaccuracy at $100 \%$ of the robot rated velocity is $4.5 \mathrm{~mm}$. The maximum pose orientation inaccuracy is found to be 14 degrees and the maximum path orientation inaccuracy is 5 degrees. Local positional errors manifest pronounced dependence on the position of the robot end effector in the working envelope. The uncertainties of the measurements are discussed and deemed to be caused by the tool centre point calibration, the reference coordinate system transformation and the low accuracy of the photogrammetry system.
\end{abstract}

\section{INTRODUCTION}

Compared to manual quality inspections, the combination of Non-Destructive Testing (NDT) and metrology through robotic deployment can offer an improvement in accuracy, precision and speed of inspection while reducing time and associated labour costs. Traditionally, programming of the robot movements required manual jogging of the end effector to specific points on the path. More recently, OffLine Path-Programming (OLP) has become common, enhancing the programming flexibility and reducing the downtime [1-3]. Moreover, OLP helps to maintain a controlled orientation of the inspection tool (e.g. a laser probe or an NDT transducer) with respect to the scanned surface, and thus further improve inspection accuracy.

However, off-line generated scan paths are not immune to errors, due to part deviation from its CAD model and deviations of robot characteristics from their kinematic model (tolerances in robot linkages, elasticity in the robot arm, encoder resolution, and lack of repeatability during calibration) [4]. Therefore, in order to improve the accuracy of an industrial robot, calibrations are required to determine the actual values of kinematic and dynamic parameters of the robot [5]. Robot calibration leads to a higher absolute positioning accuracy (a better correspondence between the real positions of the robot end effector and the positions calculated from the mathematical model of the robot).

\footnotetext{
* This work was supported by EPSRC, EP/G038627/1, "New Imaging Systems for Advanced Non-Destructive Evaluation", and EP/F017332/1, UK Research Centre in NDE. The authors are thankful to Hexagon Metrology for loan of T-Mac 6 DoF laser tracker equipment. This publication is supported by multiple datasets, which are openly available at locations cited in the reference section.

All authors are with the University of Strathclyde, Department of Electronic and Electrical Engineering, Centre for Ultrasonic Engineering, Glasgow, UK (corresponding author: Jonathan Riise; phone: +44 (0)1639 873 148; fax: +44 (0)141 548 2950; e-mail: jonathan.riise@ strath.ac.uk).
}

Absolute positioning accuracy is particularly relevant to allow robot exchangeability and OLP for precision applications [6]. Besides overall robot calibration, the calibration of its tools can minimize inaccuracies and improve process reliability. Robot calibration requires the measurement of a robot's poses and path errors; they can be carried out through a variety of methods including laser interferometry, photogrammetry, ultrasonic range finders etc.

This paper presents quantitative accuracy results of a sixaxis hollow wrist robotic arm, KUKA KR5 arc HW [7], which is known to be less accurate than other robots due to the hollow wrist characteristics.

\section{Pose AcCuracy Measurements}

\section{a. Experimental setup}

This subsection represents a study of pose accuracy of the KR5 robot as a function of the static position of the arm within its work envelope. A Leica Absolute Tracker (LAT) AT901-LR 6 DoF (Degrees of Freedom) system [8] was employed to provide ground truth through acquiring poses (coordinates and orientations) of a Leica T-MAC probe mounted to the wrist of the robot. The Leica T-MAC (Tracker-Machine Control sensor) is a 6 DoF tracking device for automated applications. The T-MAC was calibrated as a robot tool in the KUKA control system such that the Tool Centre Point (TCP) was defined to coincide with the reflector positioned at the center of the T-MAC - a Tooling Ball Reflector (TBR) (a sphere reflector of 0.5 inch diameter). Fig. 1 shows the layout of the experimental setup.

The pose and path accuracies depend on the accuracy of the TCP calibration. The purpose of a TCP calibration is to determine the offset and orientation of the tool end point with respect to the robot flange reference system.

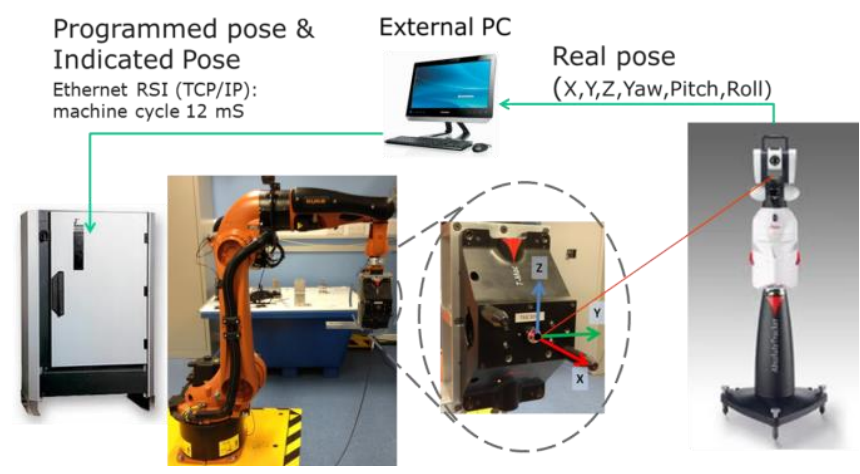

Figure 1. Calibration process through laser tracker, showing (from left to right): robot controller, KUKA KR5 arc HW with T-Mac, LAT AT901.

The most common TCP calibration procedure consists in the 4 point method, based on exploiting the tip of a solid 
spike as reference point and bringing the robot tool to this point from four sufficiently different directions. Since the TMAC sensor reflector is positioned in the middle of the device, the standard 4 point TCP calibration procedure cannot be used. Therefore, a "laser spike" procedure was used; the coordinates of the first (reference) position of the TCP (reflector) were measured by the LAT and annotated. Then, the robot was manually jogged to bring the reflector to the same point from three different directions. The TCP coordinates were continuously compared with the reference values until they coincided (within a set tolerance of $100 \mu \mathrm{m}$ ). The resulting TCP calibration uncertainty was $0.32 \mathrm{~mm}$.

The LAT and robot reference systems do not coincide. In order to evaluate the accuracy of a moving TCP, the coordinates reported by the laser tracker need to be transformed to the robot reference system. For this purpose, the robot TCP was moved to three different points in the robot working envelope. The respective coordinates were measured by both the laser tracker and the robot positional feedback and the transformation matrix was determined. The transformation of the coordinates from the reference system of the absolute laser tracker to the global coordinate frame of the robot was carried out by the following homogeneous transformation [9]:

$$
\left(\begin{array}{c}
\boldsymbol{r}_{j} \\
1
\end{array}\right)=\left(\begin{array}{cc}
\boldsymbol{R}_{i, j} & \boldsymbol{p}_{i, j} \\
\mathbf{0}^{\mathrm{T}} & 1
\end{array}\right)\left(\begin{array}{c}
\boldsymbol{r}_{i} \\
1
\end{array}\right),
$$

where $\boldsymbol{r}_{i}$ and $\boldsymbol{r}_{j}$ are the position vectors in the LAT and robot coordinate frames, respectively, $\boldsymbol{R}_{i, j}$ is the rotation matrix of the LAT frame $i$ relative to the robot frame $j$ and $\boldsymbol{p}_{i, j}$ is the translation vector of the frame origin.

\section{b. Positional accuracy}

The ISO 9283:1998 standard [10], prescribes specific poses for robot accuracy measurements. The standard states that a diagonal plane of a cube, contained in the space of interest of the robot working envelope, should be used to determine the accuracy and the repeatability of the robot. A robot program for this experiment was designed using the $K U K A \mid P R C$ [11] software add-on in the Grasshopper plugin [12] of the Rhinoceros $5 \mathrm{CAD}$ package. The path contained five command points and the robot repeated the same path 30 times in accordance with the standard. The path is shown in Fig. 2a. The idea behind this approach is to test the robot repeatability as well as the accuracy by visiting the same positions a number of times. The LAT and the motion capture system Vicon T160 [13] were used in the experiment.

A special end effector was designed to carry out the test. The end effector contained one TBR reflector for the LAT and 4 Vicon markers placed at known distances from the central marker, as shown in Fig. 2b. The LAT was used to provide a ground truth, as this system is certified to have an accuracy of $\pm 10 \mu \mathrm{m}$ at a distance of $4 \mathrm{~m}$. A virtual Vicon tracking object was created with its center coincident with the Leica reflector. An average of 100 readings was taken for each robot pose.

The measured coordinates obtained from the 30 repetitions of the same path and transformed to the same reference system are shown in Fig. 3. The Euclidean distances between the points were compared. The obtained results are illustrated in Fig. 4. The spread is defined as the sphere centred on the barycentre of the measurements, with a radius equal to the average distance of the measurements from the center. The semi-transparent sphere helps to visualize the accuracy and precision of the robot internal measurement system and of the Vicon system. Each plot also contains the vectorial difference between observed and commanded points.

There is a significant difference between the Leica measurement and the center of the Vicon virtual tracking object. The pose positioning accuracy is calculated as:

$A P_{P}=\sqrt{\left(\bar{x}-x_{c}\right)^{2}+\left(\bar{y}-y_{c}\right)^{2}+\left(\bar{z}-z_{c}\right)^{2}}$,
$A P_{x}=\left(\bar{x}-x_{c}\right), \quad A P_{y}=\left(\bar{y}-y_{c}\right), \quad A P_{z}=\left(\bar{z}-z_{c}\right)$,
$\bar{x}=\frac{1}{n} \sum_{j-1}^{n} x_{j}, \quad \bar{y}=\frac{1}{n} \sum_{j-1}^{n} y_{j}, \quad \bar{z}=\frac{1}{n} \sum_{j-1}^{n} z_{j}$,

where $\bar{x}, \bar{y}$ and $\bar{z}$ are the coordinates of the barycentre of the cluster of points obtained after repeating the same pose $n$ times, $x_{c}, y_{c}$ and $z_{c}$ are the coordinates of the command pose and $\mathrm{x}_{\mathrm{j}}, \mathrm{y}_{\mathrm{j}}$ and $\mathrm{z}_{\mathrm{j}}$ are the coordinates of the $j^{\text {th }}$ attained pose.

As evident from the data presented in Table 1 the internal positioning system of the robot is more accurate than the Vicon T160 system, for the used configuration. As mentioned earlier, and as it is demonstrated in the literature, the accuracy of the Vicon system is strongly affected by the placement of the cameras as well as by the distance between the tracking objects and the cameras. The large difference in accuracy between pose 2 and pose 4 in the Vicon system indicates that the accuracy of the system is highly dependent on the position of the markers in relation to the cameras.

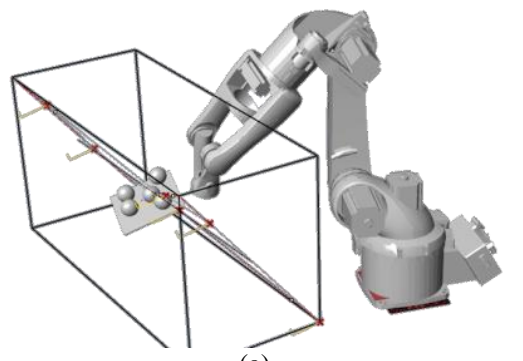

(a)

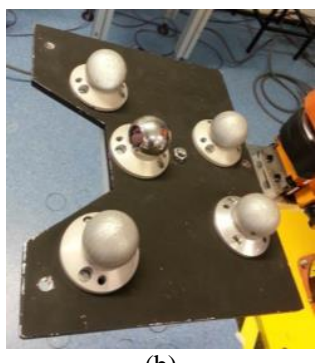

(b)
Figure 2. a) Robotic path for pose precision and accuracy study according to ISO 9283:1998. b) Plate attached to robot with one LAT reflector and 4 Vicon tracking objects.

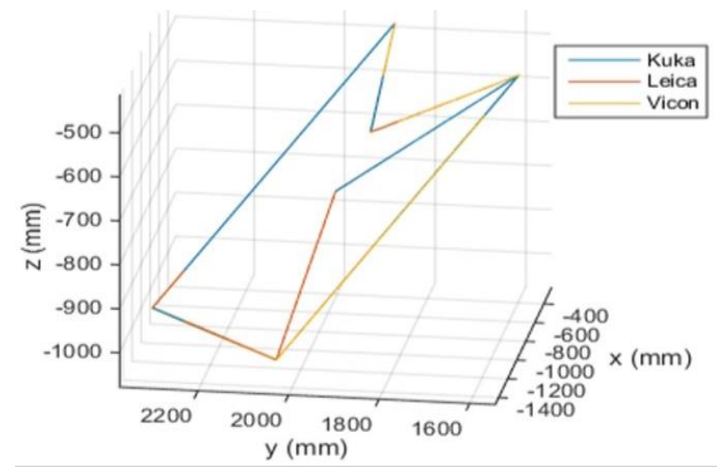

Figure 3. Data acquired using LAT, Vicon and robot encoders. 


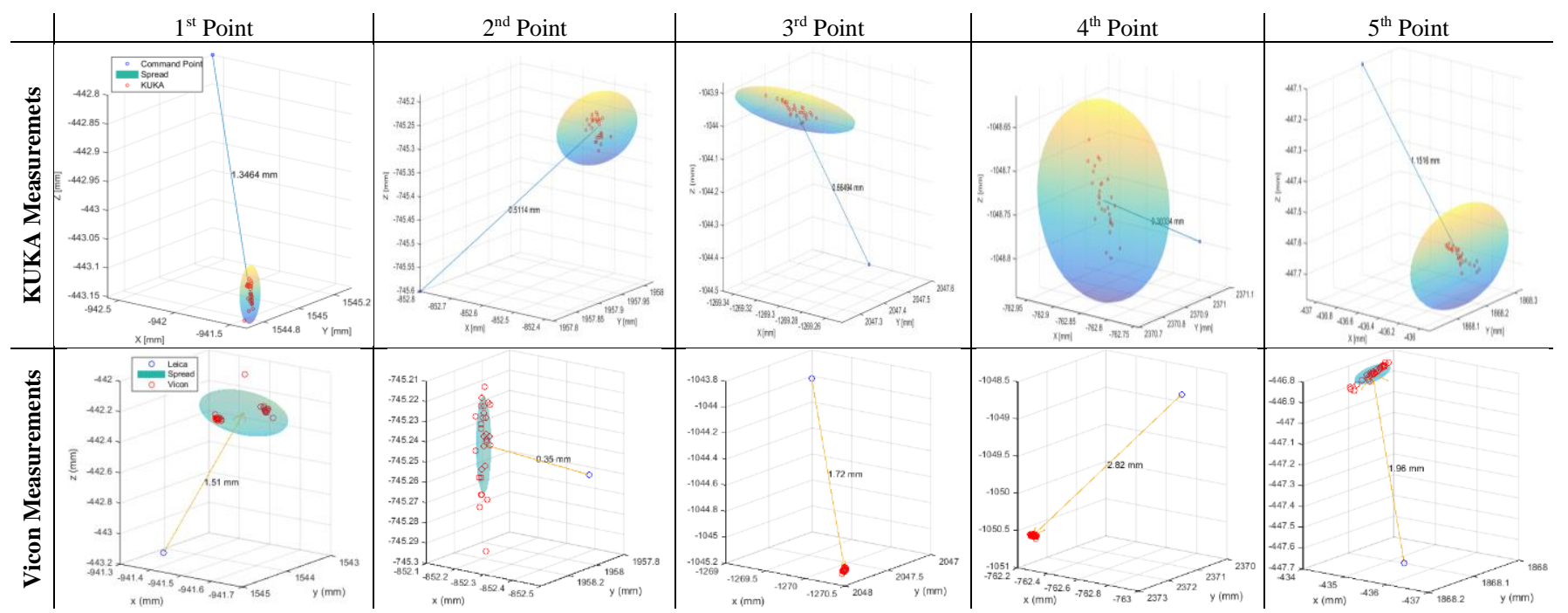

Figure 4. KUKA controller's measurements (first row) and Vicon measurements (second row), compared to the observed LAT points.

TABLE I. ACCURACY AND REPEATABILITY OF KUKA AND VICON MEASUREMENTS, ACCORDING TO ISO9283:1998.

\begin{tabular}{|l|l|l|l|l|l|}
\hline \multicolumn{3}{|c|}{ KUKA } & \multicolumn{3}{c|}{ VICON } \\
\hline Pose & $\begin{array}{l}\text { Accuracy } \\
(\mathbf{m m})\end{array}$ & $\begin{array}{l}\text { Repeatability } \\
(\mathbf{m m})\end{array}$ & Pose & $\begin{array}{l}\text { Accuracy } \\
(\mathbf{m m})\end{array}$ & $\begin{array}{l}\text { Repeatability } \\
(\mathbf{m m})\end{array}$ \\
\hline 1 & 1.3464 & 0.0472 & 1 & 1.5100 & 0.1448 \\
\hline 2 & 0.5114 & 0.0522 & 2 & 0.3500 & 0.0240 \\
\hline 3 & 0.6649 & 0.0452 & 3 & 1.7200 & 0.0156 \\
\hline 4 & 0.3033 & 0.0847 & 4 & 2.8200 & 0.0180 \\
\hline 5 & 1.1516 & 0.1193 & 5 & 1.9600 & 0.0400 \\
\hline Aver. & 0.7955 & 0.0697 & Aver. & 1.6720 & 0.0485 \\
\hline
\end{tabular}

The experimental repeatability of the robot positions is only slightly worse than the manufacturer's specification (stated as $0.04 \mathrm{~mm}$ ). Certain poses can cause higher loads on the joints due to the weight of the robot which can lead to deviations in positional accuracy. Although the robot positional feedback is better at returning accurate positions in such situations, the repeatability of the Vicon system is on average better. The two systems can be respectively described as being accurate but less precise (robot feedback) and inaccurate but precise (Vicon). To gain a better understanding of how these positional errors were distributed over the working envelope of the robot, a new experiment was carried out with a different toolpath.

Theoretically, the ISO 9283:1998 approach is designed to be fast and representative of the robot's pose accuracy. However, the prescribed poses lie on a single plane. A longer test was performed in a wider working envelope volume. The robot's TCP followed the discrete rectangular path shown in Fig. 5. The origin of the plot corresponds to the origin of a reference system with coordinates: $X=200, Y=-500, Z=650$ and $A=B=C=0$. The robot paused at every point of the path and the LAT acquired 333 readings, which were averaged. The robot command and feedback coordinates were transmitted via an Ethernet socket, established through KUKA add-on software called Robot Sensor Interface (RSI).

Fig. 6 represents dependences of errors of every coordinate component on the path. The large off-set most probably correlates with the TCP calibration error. All components show distinguishable cyclic behavior, with that of $\Delta X$ being most pronounced. The two most prominent periods might be due to the fact that the $\mathrm{Y}$ component changed symmetrically from $-500 \mathrm{~mm}$ to $+500 \mathrm{~mm}$ (relatively to the robot global reference system) and the laser tracker was approximately positioned along the global $\mathrm{x}$-axis of the robot. The deviations obtained using the laser tracker with respect to the actual robot coordinates are significant, going up to $1.6 \mathrm{~mm}$.

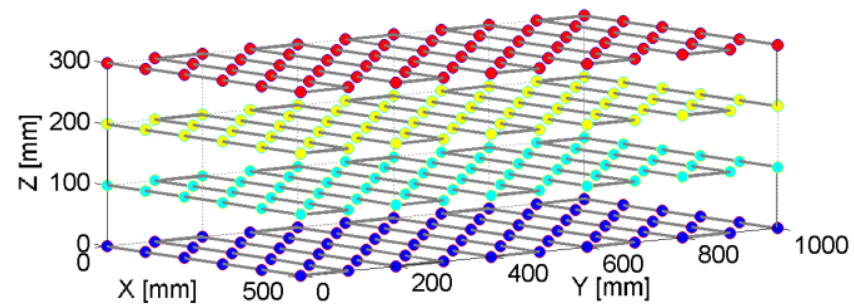

Figure 5. Rectangular box scan path.

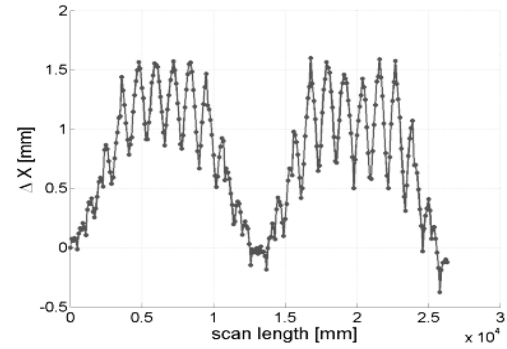

(a)

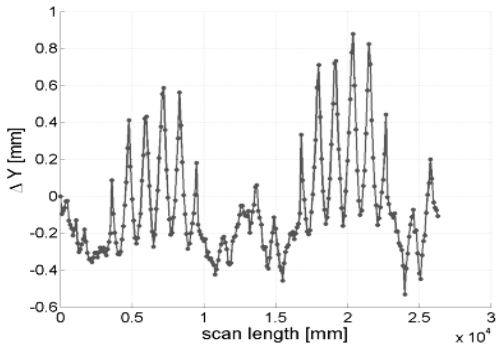

(b)

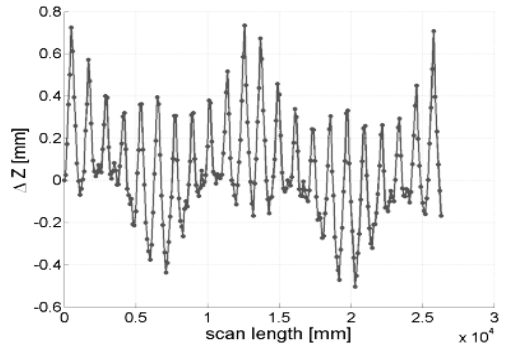

(c)

Figure 6. Dependence position error of on scan path: (a) X, (b) $\mathrm{Y}$, (c) $\mathrm{Z}$ coordinates. 


\section{c. Orientation accuracy}

This subsection focuses on the study of the orientation accuracy of a KUKA KR5 arc HW robot. As in the previous subsection, the LAT and the T-MAC sensor were used. The robot's TCP was rotated about the $\mathrm{x}, \mathrm{y}$, and $\mathrm{z}$-axis in increments of 10 degrees. At every pose the robot stopped and the laser tracker acquired 333 readings, which were averaged. The LAT coordinates were transformed to the robot's frame using equation (1). Six robot poses, with different distances from the center of the envelope, were studied.

The TCP was programmed to stay stationary in one point. However, as it can be observed from Fig. 7a, the XYZ coordinates acquired by the LAT show that the TCP loci form a spherical shell segment with radius of approximately equal to $5.6 \mathrm{~mm}$. The extent of the TCP dispersion is deemed to be due to multiple factors (e.g. the error of the TCP calibration and deviation of robot kinematic characteristics). The point cloud has the largest extent in YZ plane since the principal rotation was carried out about the $\mathrm{x}$-axis, corresponding to robot roll angle $\mathrm{C}$, which changed from -90 deg. to +90 deg. (see Fig. 7b).

Fig. 8a shows an average map of angle $C$ in $A B$ plane. This is a saddle function, given by $\Delta \mathrm{C}(\mathrm{A}, \mathrm{B}) \cong-k \cdot \Delta \mathrm{A} \cdot \Delta \mathrm{B}$, where $k$ is a constant coefficient. LAT data were not acquired at the corner given by $\mathrm{A}=-30, \mathrm{~B}=20$, since the acceptance angle of reflector was exceeded; thus, these data were replaced by KUKA actual coordinates and orientations. Fig. 8b presents one of the averaged C columns shown in Fig. $7 \mathrm{~b}$. The $\mathrm{ABC}$ orientation acquired by the laser tracker produces a semi-helical locus around the commanded robot orientation. The relationship is given by $(\Delta \mathrm{A}, \Delta \mathrm{B}, \Delta \mathrm{C}) \cong$ $[\rho \cdot \sin (\mathrm{C}), \rho \cdot \cos (\mathrm{C}), \mathrm{C}]$, where $\rho \approx 1.4$.

Fig. 9 represents the dependence of the error for every TCP coordinate component on the integral rotational path. As it was discussed above, the principal rotation was carried out about axis X (roll C) and the TCP loci formed a spherical shell segment with radius of approximately $6 \mathrm{~mm}$ (see Fig. 7a). Therefore, the $\mathrm{Y}$ and $\mathrm{Z}$ coordinates exhibit more pronounced harmonic behaviour, with the number of periods of $\Delta Y$ being 36 , which corresponds to 6 cycles of yaw angle A times 6 cycles of pitch angle B. The number of periods of $\Delta \mathrm{Z}$ is 18 , which corresponds to the number of steps between discrete $\mathrm{C}$ orientations. Maximum absolute pose orientation inaccuracy occurs in the roll angle and it is circa 14 degrees.

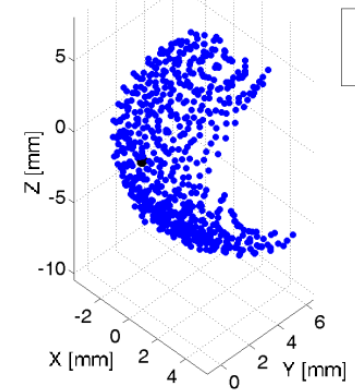

(a)

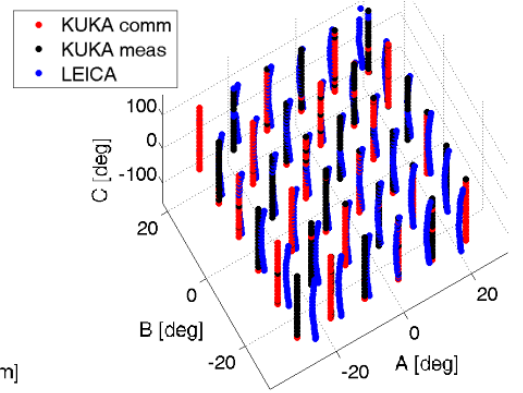

(b)
Figure 7. a) XYZ coordinates resulting from TCP rotation; b) Orientation angles of TCP rotation.

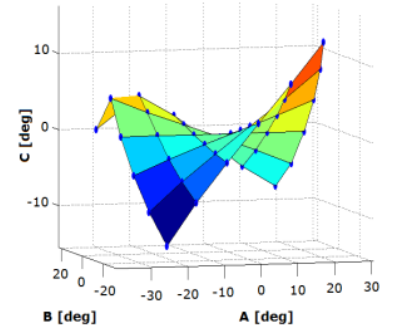

(a)

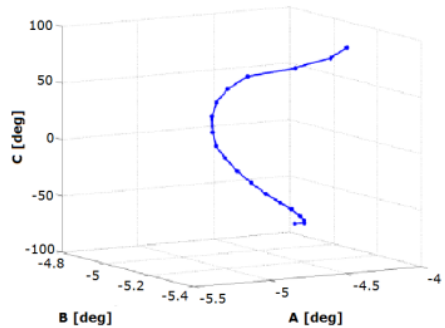

(b)
Figure 8. Orientation angles of TCP rotation in pose 1: (a) 3D, (b) AB plane projection.
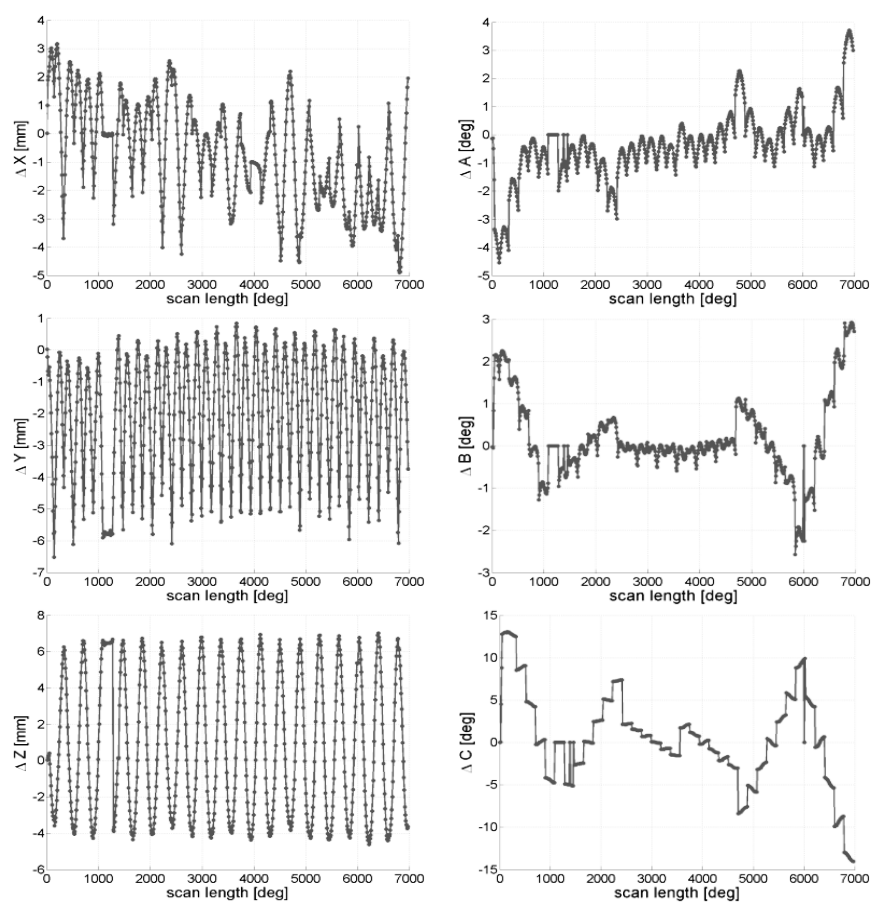

Figure 9. Dependence of position (X,Y and $\mathrm{Z}$ components) and orientation (yaw, pitch and roll angles) error on the integral rotation.

\section{PATH ACCURACY CHARACTERISATION}

Path accuracy is an important parameter of any robotic quality inspection, especially when using OPL. The ISO 9283:1998 [10] defines the path accuracy as the maximum path deviation along the path, in terms of position and orientation. The positioning path accuracy is calculated as:

$A T_{P}=\max \sqrt{\left(\bar{x}_{i}-x_{c i}\right)^{2}+\left(\bar{y}_{i}-y_{c i}\right)^{2}+\left(\bar{z}_{i}-z_{c i}\right)^{2}}$,

where $x_{c i}, y_{c i}$ and $z_{c i}$ are the coordinates of the $i$-th point on the command path and $\overline{\mathrm{x}}_{\mathrm{i}}, \overline{\mathrm{y}}_{\mathrm{i}}$ and $\overline{\mathrm{z}}_{\mathrm{i}}$ are the corresponding coordinates of the $i$-th barycenter of the attained path.

The orientation path accuracies $A T_{a}, A T_{b}$ and $A T_{c}$ are defined as the maximum deviations from commanded rotations around the $\mathrm{x}, \mathrm{y}$ and $\mathrm{z}$-axis along the path, respectively:

$A T_{a}=\max \left|\bar{a}_{i}-a_{c i}\right|$,

where $a_{c i}$ is the command orientation at the point and $\bar{a}_{i}$ is the average attained orientation angle at the $i$-th point of the path. Expressions similar to (6) are used for $A T_{b}$ and $A T_{c}$. 
First, the path accuracy of the robot was measured according to ISO 9283:1998. Since the positional feedback of the robot encoders can be inaccurate due to deviations of the kinematic parameters [14], the LAT was used as an external measurement system. The available LAT at the time of testing was only capable of three DoF measurements. ISO 9283:1998 prescribes four alternative paths which can be used to characterize a robot's path accuracy: a straight line, a rectangular path and two circular paths. The rectangular path was chosen since it was the most morphologically similar path to the NDT path used to scan the leading edge skin panel shown in Fig. 11a. As prescribed by [10], the robot performed 10 cycles, moving at three override speeds: $10 \%$, $50 \%$ and $100 \%$ of the maximum robot rated speed. The LAT sampled the position of a TBR mounted at the robot TCP, with a sampling frequency of $1 \mathrm{kHz}$. The robot command positions and the feedback coordinates were transmitted, once again, via the Ethernet RSI interface. The data from the robot's controller and from the LAT controller were synchronized through purposely developed data acquisition software using a single PC to timestamp all incoming data packets. Fig. 10 gives the path points measured at $100 \%$ of the rated speed, showing command and feedback positions as well as the points measured through the LAT. Table 2 presents the resulting path accuracy for three robot speeds. As expected, the error increases with the speed. After having determined the path accuracy of the robot along a path prescribed by ISO 9283:1998, accuracy investigations were carried out on the robot path generated for the inspection of the leading edge sample.

TABLE II. PATH ACCURACY ACCORDING TO ISO 9283:1998

\begin{tabular}{lll}
\hline$@ \mathbf{1 0 \%}$ speed & $@ \mathbf{5 0 \%}$ speed & @100\% speed \\
\hline $1.844 \mathrm{~mm}$ & $3.178 \mathrm{~mm}$ & $4.464 \mathrm{~mm}$ \\
\hline
\end{tabular}

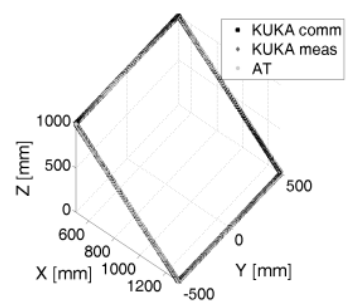

Figure 10. Shape of the test path as measured at $100 \%$ rated speed showing command and feedback positions as well as the LAT measured coordinates.

(a)

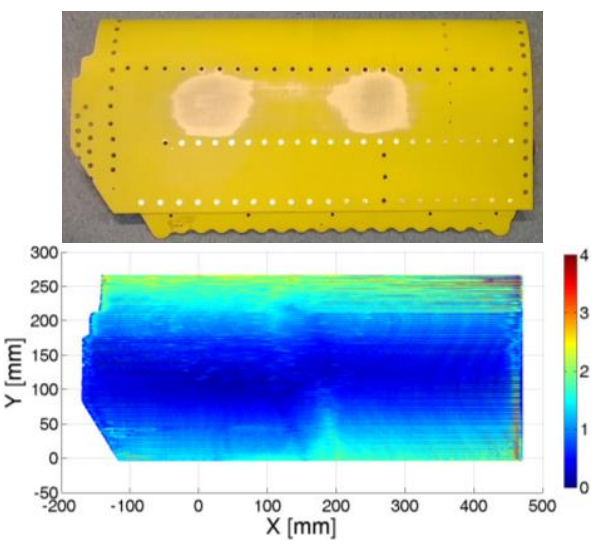

Figure 11. (a) Leading edge skin panel sample and (b) positional error of the generated inspection scan path at $100 \%$ of the rated robot speed.
Fig. 11b shows the resulting distribution map of the positional error (in $\mathrm{mm}$ ) at $100 \%$ of the rated robot speed. Component-wise, the major positional error is exhibited in the scan direction of the raster path. The $x$ component has the biggest error along the passes and $y$ component has the biggest error when the robot moves between the passes, which results in larger errors at the scan area edges. Table 3 presents the resulting accuracy for the scan path. The positional accuracy of continuous motion inversely depends on the motion speed; the faster the motion the bigger the positional errors. In order to compensate for the pose error, the output of the laser tracker or other metrological system can be used to produce real-time robot path-correction through the RSI add-on. The accuracy values presented in Table 3 are somewhat higher than the corresponding values in Table 2, which can be due to the fact that the NDT scan pass has a significantly smaller extent than the path prescribed by [10]. The scanned part was also located closer to the central area of the working envelope of the robot, whereas bigger inaccuracies can be expected at the extremes of the robot's reach. Thus the NDT scan path is not representative of the robot accuracy over the entire working envelope.

The standard deviation of the path attained at $10 \%$ of the rated velocity (suitable for NDT inspection) was calculated to be below $0.35 \mathrm{~mm}$. Considering the errors resulting from the laser mapping of the leading edge skin panel and the error due to TCP calibration, the positional uncertainty of the NDT scan does not exceed $0.5 \mathrm{~mm}$.

In addition to the path prescribed by the ISO standard, an arbitrary path was tested. The robot's TCP was steadily moved in 3D space and rotated about ABC angles. Fig. 12 shows the programmed path and the orientation angles. Fig. 13 shows the differences between the scan path coordinates measured by robot and by the LAT. It is remarkable that $\Delta \mathrm{Y}$ features a second harmonic modulation (probably due to double frequency of variation of angle $\mathrm{A}$ ), but $\Delta \mathrm{Z}$ features only a single harmonic modulation. It might be explained by the $\Delta \mathrm{Z}$ dependence on angular scan, which correlates with changes of angle $\mathrm{C}$, as shown in section $2 \mathrm{c}$ ).

Fig. 14 gives the commanded path angular coordinates. Fig. 15 shows differences between the path orientation angles measured by robot and laser tracker. The second harmonic modulation of $\Delta \mathrm{B}$ and $\Delta \mathrm{C}$ due to double frequency of variation of $\mathrm{A}$ is remarkable.

TABLE III. PATH ACCURACY MEASURED ALONG THE NDT SCAN PATH

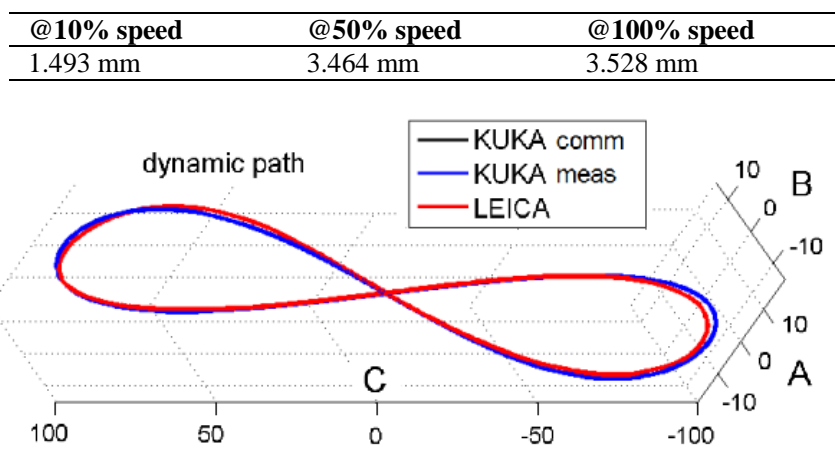

Figure 12. Arbitrary path and orientation angles in 3D. 


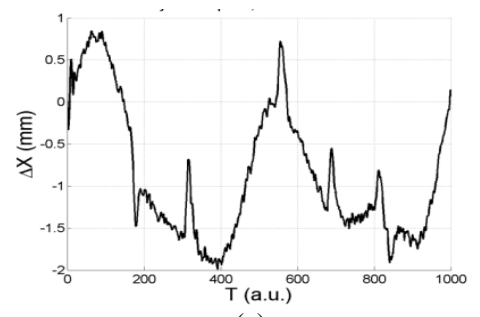

(a)

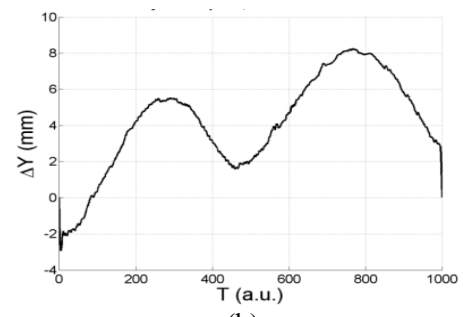

(b)

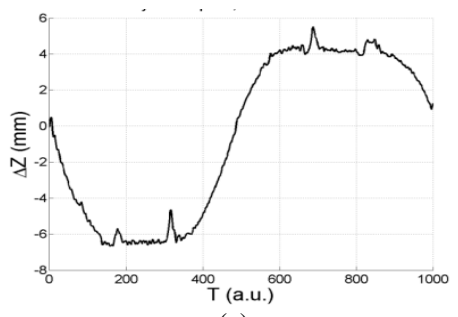

(c)

Figure 13. Differences between scan path coordinates measured by robot and laser tracker: (a) $\Delta \mathrm{X}$, (b) $\Delta \mathrm{Y},(\mathrm{c}) \Delta \mathrm{Z}$.

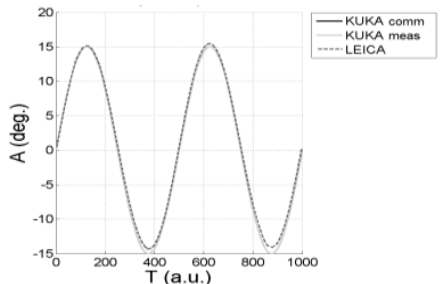

(a)

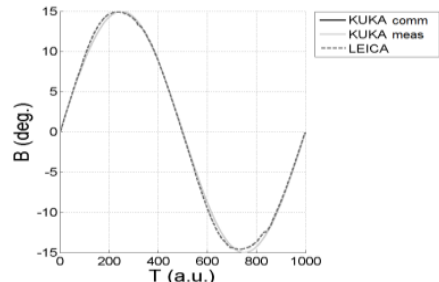

(b)

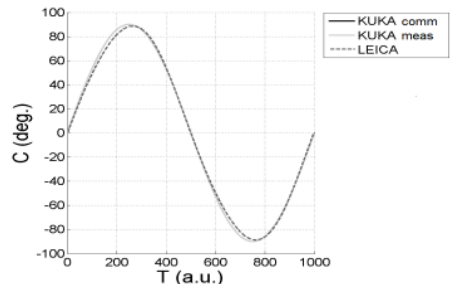

(c)

Figure 14. Scan path orientation angles: (a) A, (b) B, (c) C.

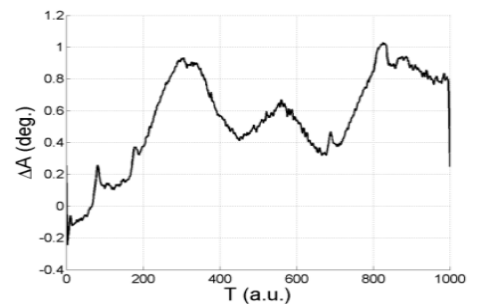

(a)

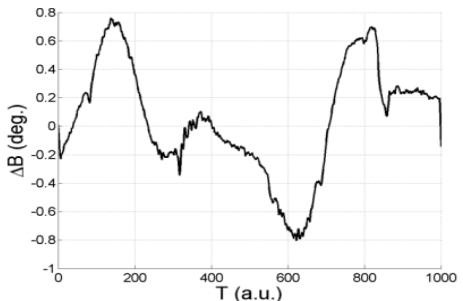

(b)

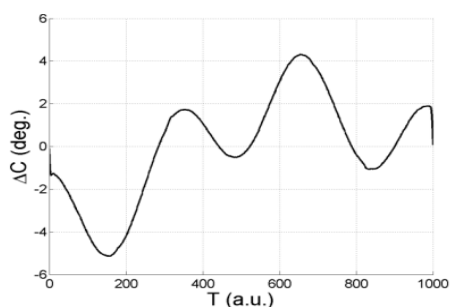

(c)

Figure 15. Differences between scan path orientation angles measured by robot and laser tracker: (a) $\Delta \mathrm{A}$, (b) $\Delta \mathrm{B}$, (c) $\Delta \mathrm{C}$.

\section{CONCLUSION}

An accuracy and repeatability study of a KUKA KR5 arc HW robot has been conducted using both laser tracking and large volume photogrammetric methods. The study has been undertaken to determine both static and dynamic path accuracies according to ISO 9283:1998, two factors which are crucial to performing automated quality inspections of complex geometry components. It was found that the overall positioning inaccuracy was almost $1 \mathrm{~mm}$ and the path inaccuracy at maximum robot velocity was $4.5 \mathrm{~mm}$. The largest pose orientation inaccuracy was found to be 14 degrees. Variations in positioning and path inaccuracies show a marked dependence on the robot joint configuration and deteriorate towards the edges of the working envelope of the robot. The uncertainty of a robotic inspection application was estimated to be within $0.5 \mathrm{~mm}$ which is sufficient for most automated quality assurance tasks. Uncertainties arising from robotic tool calibration, reference system transformations and low accuracy of the photogrammetry system were identified.

Our future work will aim at implementing real-time robot path-correction of the path trajectories, based on metrological feedback.

\section{REFERENCES}

C. Mineo, S. G. Pierce, B. Wright, I. Cooper, and P. I. Nicholson, "PAUT inspection of complex-shaped composite materials through six DOFs robotic manipulators," Insight: NonDestructive Testing and Condition Monitoring, vol. 57, pp. 161166, 2015.
[2] G. Wittenberg, "Developments in offline programming: An overview," Industrial Robot, vol. 22, pp. 21-23, 1995.

[3] S. Carter, "OFF-LINE ROBOT PROGRAMMING: THE STATE-OF-THE-ART," Industrial Robot, vol. 14, pp. 213-215, 1987.

[4] E. Roos and A. Behrens, "Off-line programming of industrial robots - Adaptation of simulated user programs to the real environment," Computers in Industry, vol. 33, pp. 139-150, 1997.

[5] T. Messay, R. Ordóñez, and E. Marcil, "Computationally efficient and robust kinematic calibration methodologies and their application to industrial robots," Robotics and ComputerIntegrated Manufacturing, vol. 37, pp. 33-48, 2016.

[6] K. Young, C.G. Pickin, " Accuracy assessment of the modern industrial robot," The Industrial Robot, vol. 27, pp 427-436, 2000.

[7] KUKA. KR5 arc $H W$ datasheet. Available: http://www.kukarobotics.com/res/sps/f776ebab-f613-4818-9feb527612db8dc4 PF0012 KR 5 arc HW en.pdf

[8] Leica_Geosystems, "AbsoluteTracker, User Manual," ed.

[9] Springer Handbook of Robotics: Springer-Verlag Berlin Heidelberg, 2008.

[10] "ISO 9283:1998(en) Manipulating industrial robots Performance criteria and related test methods," ed.

[11] Association for Robots in Architecture. "KUKA|PRC". Available: http://www.robotsinarchitecture.org/kuka-prc

[12] "Grasshopper - Algorithmic Modelling for Rhino." Available: http://www.grasshopper3d.com/

[13] Vicon. Available: http://www.vicon.com/

[14] M. Slamani, A. Nubiola, and I. Bonev, "Assessment of the positioning performance of an industrial robot," Industrial Robot, vol. 39, pp. 57-68, 2012.

Dataset for Section II(a-b) is openly available at: http://dx.doi.org/10.15129/8b4f29a0-4985-4281-9677-8bf73991ab6f 